\title{
NONASSOCIATIVE RINGS WITH A SPECIAL DERIVATION
}

\author{
CHEN-TE YEN
}

\begin{abstract}
Let $R$ be a nonassociative ring, $N, L$ and $G$ the left nucleus, right nucleus and nucleus respectively. It is shown that if $R$ is a prime ring with a derivation $d$ such that $a x+d(x) \in G$ where $a \in \mathbb{Z}$, the ring of rational integers, or $a \in G$ with $(a d)(x)=a d(x)=d(a x)$ and $a x=x a$ for all $x$ in $R$ then either $R$ is associative or $a d+d^{2}=2 d(R)^{2}=0$. This result is also valid under the weaker hypothesis $a x+d(x) \in N \cap L$ for all $x$ in $R$ for the simple ring case, and we obtain that either $R$ is associative or $\left(\left(a d+d^{2}\right)(R)\right)^{2}=0$ for the prime ring case.
\end{abstract}

\section{Introduction}

Let $R$ be a nonassociative ring. We adopt the usual notation for associators : $(x, y, z)=(x y) z-x(y z)$. We shall denote the left nucleus, middle nucleus, right nucleus and nucleus by $N, M, L$ and $G$ respectively. Thus $N, M, L$ and $G$ consists of all elements $n$ such that $(n, R, R)=0,(R, n, R)=0,(R, R, n)=0$ and $(n, R, R)=$ $(R, n, R)=(R, R, n)=0$ respectively. An additive mapping $d$ on $R$ is called a derivation if $d(x y)=d(x) y+x d(y)$ for all $x, y$ in $R$. $R$ is called semiprime if the only ideal of $R$ which squares to zero is the zero ideal. $R$ is called prime if the product of any two nonzero ideals of $R$ is nonzero. $R$ is called simple if $R$ is the only nonzero ideal of $R$. Clearly, a prime ring is a semiprime ring. If $R$ is a simple ring, then $R^{2}=0$ or $R^{2}=R$; in the former case $R$ is commutative and associative. So, if $R$ is a simple ring then we assume that $R^{2}=R$. Thus a simple ring is a prime ring. Recently, Suh [3] proved that if $R$ is a prime ring with a derivation $d$ such that $d(R) \subseteq G$ then either $R$ is associative or $d^{3}=0$. We generalized and improved Suh's results as follows:

Theorem $\mathbb{A}$ [6]. If $R$ is a prime ring with a derivation $d$ such that $d(R) \subseteq$ $N \cap M$ or $d(R) \subseteq M \cap L$ or $d(R) \subseteq N \cap L$, then either $R$ is associative or $d^{2}=2 d=0$.

Received June 30, 1993, revised October 14, 1993, revised April 19, 19995.

1991 Mathematics Subject Classification. Primary 17A36.

Key words and phrases. Nonassociative ring, nucleus, derivation, semiprime ring, prime ring, simple ring, $d$-invariant. 
Theorem B [8]. If $R$ is a prime ring with a derivation $d$ and there exists a fixed positive integer $n$ such that $d^{n}(R) \subseteq G$, then either $R$ is associative or $d^{3 n-1}=0$.

In [7], using the result of [2], we have partially extended Theorem A. The purpose of this note is to prove that if $R$ is a prime ring with a derivation $d$ such that $a x+d(x) \in G$ for all $x$ in $R$ where $a$ is as in the Abstract then either $R$ is associative or $a d+d^{2}=2 d(R)^{2}=0$. This result is also valid under the weaker hypothesis $a x+d(x) \in N \cap L$ for all $x$ in $R$ for the simple ring case, and we obtain that either $R$ is associative or $\left(\left(a d+d^{2}\right)(R)\right)^{2}=0$ for the prime ring case. Rings with associators in the nuclei were first studied by Kleinfeld and later by the author. Kleinfeld [1] showed that if $R$ is a semiprime ring such that $(R, R, R) \subseteq G$ and the Abelian group $(R,+)$ has no elements of order 2 then $R$ is associative. In [4], we proved that if $R$ is a simple ring of characteristic not two such that $(R, R, R) \subseteq N \cap M$ or $(R, R, R) \subseteq M \cap L$ then $R$ is associative. In [5], we have proved that if $R$ is a semiprime ring such that $(R, R, R) \subseteq N \cap M$ or $(R, R, R) \subseteq M \cap L$ or $(R, R, R) \subseteq N \cap L$ then $N=M=L$ and $2(R, R, R)=0$. Thus $E$. Kleinfeld's result can be improved.

Let $R$ be a ring with a derivation $d$. A nonempty subset $S$ of $R$ is called $d$-invariant if $d(S) \subseteq S$.

\section{Results}

Let $R$ be a nonassociative ring. In every ring one may verify the Teichmüller identity

$$
(w x, y, z)-(w, x y, z)+(w, x, y z)=w(x, y, z)+(w, x, y) z \quad \text { for all } w, x, y, z \text { in } R .
$$

Suppose that $n \in N$. Then with $w=n$ in (1) we obtain

$$
(n x, y, z)=n(x, y, z) \text { for all } n \text { in } N, \quad \text { and all } x, y, z \text { in } R \text {. }
$$

Assume that $m \in L$. Then with $z=m$ in (1) we get

$$
(w, x, y m)=(w, x, y) m \text { for all } m \text { in } L, \quad \text { and all } w, x, y \text { in } R \text {. }
$$

As consequences of (1), (2) and (3), we have that $N, M, L, N \cap M, M \cap L, N \cap L$ and $G$ are associative subrings of $R$.

Definition 1. Let $d$ be a derivation of $R$, and $a \in \mathbb{Z}$, the ring of rational integers, or $a \in G$ with $(a d)(x)=a d(x)=d(a x)$ and $a x=x a$ for all $x$ in $R$.

We assume that $R$ has a derivation $d$ which satisfies

$$
a x+d(x) \in A \text { for all } x \text { in } R \text {, where } A \text { is a subring of } R \text {. }
$$


Assume that $x, y \in R$. Using the definition of $d$, Definition 1 and (*), and noting that $A$ is a subring of $R$, we get

$$
a^{2} x y+a d(x y)+d(x) d(y)=(a x+d(x))(a y+d(y)) \in A .
$$

By Definition 1 and $(*), a^{2} x y+a d(x y)=a(a(x y))+d(a(x y)) \in A$. Noting that $A$ is a subring of $R$, these two relations imply

$$
d(x) d(y) \in A \text { for all } x, y \text { in } R \text {. }
$$

Applying Definition 1 and $(*)$, we have

$$
\left(a d+d^{2}\right)(x) \cdot y+d(x) d(y)=\left(a d(x)+d^{2}(x)\right) \cdot y+d(x) d(y)=a(d(x) y)+d(d(x) y) \in A
$$

Combining this with (4) yields $\left(a d+d^{2}\right)(x) \cdot y \in A$. Thus we obtain

$$
\left(a d+d^{2}\right)(R) \cdot R \subseteq A
$$

By Definition 1, (*) and symmetry, we get

$$
R \cdot\left(a d+d^{2}\right)(R) \subseteq A .
$$

Using Definition 1 and $(*)$, we have $\left(a d+d^{2}\right)(x)=a d(x)+d(d(x)) \in A$. Hence we obtain

$$
\left(a d+d^{2}\right)(R) \subseteq A
$$

Definition 2. The associator ideal $I$ of $R$ is the smallest ideal which contains all associators in. $R$.

Note that I may be characterized as all finite sums of associators and right (or left) multiples of associators, as a consequence of (1). Hence we have

$$
I=\sum(R, R, R)+(R, R, R) R=\sum(R, R, R)+R(R, R, R) .
$$

Lemma 1. Let $R$ be $a$ ring and $B$ an additive subgroup of $(R,+)$. If $B \subseteq G$ and $B R+R B \subseteq N \cap M$ or $M \cap L$ or $N \cap L$, then the ideal $E$ of $R$ generated by $B$ is

$$
E=\sum B+B R+R B+R \cdot B R
$$

Proof. Obviously, $E$ is an additive subgroup of $(R,+)$. By symmetry, we only prove the lemma in case $B \subseteq G$ and $B R+R B \subseteq N \cap M$. Thus we obtain

$$
(R \cdot B R) R=R \cdot(B R) R=R \cdot B R^{2} \subseteq R \cdot B R
$$


and

$$
R(R \cdot B R)=R(R B \cdot R)=(R \cdot R B) R=R^{2} B \cdot R \subseteq R B \cdot R=R \cdot B R .
$$

Hence $E$ is an ideal of $R$.

Lemma 2. If $R$ is a ring with a derivation $d$ such that $a d+d^{2}=0$, then $2 d(R)^{2}=0$.

Proof. Assume that $x, y \in R$. Because of $a d+d^{2}=0$, we obtain

$$
\begin{gathered}
-a d(x y)=d^{2}(x y)=d^{2}(x) y+2 d(x) d(y)+x d^{2}(y) \\
=-a d(x) y+2 d(x) d(y)-a x d(y)=-a d(x y)+2 d(x) d(y) .
\end{gathered}
$$

Thus $2 d(x) d(y)=0$, as desired.

Let $A \subseteq N$. Applying (2), (5) and (7), we get $\left(a d+d^{2}\right)(R) \cdot(R, R, R)=0$. By this and $(7)$, we have $\left(a d+d^{2}\right)(R) \cdot((R, R, R) R)=0$. Using these and (8), we obtain

$$
\left(a d+d^{2}\right)(R) \cdot I=0 \quad \text { if } \quad A \subseteq N .
$$

Let $A \subseteq L$. Applying (3), (6), (7) and (8), we have

$$
I \cdot\left(a d+d^{2}\right)(R)=0 \quad \text { if } \quad A \subseteq L
$$

Theorem 1. If $R$ is a simple ring with a derivation $d$ such that ax $+d(x) \in$ $N \cap L$ for all $x$ in $R$, then either $R$ is associative or ad $+d^{2}=2 d(R)^{2}=0$.

Proof. If $I=0$, then $R$ is associative. Assume that $I \neq 0$. By the simplicity of $R$, we get $I=R$. By (9) and (10), we have $\left(a d+d^{2}\right)(R) \cdot R=0$ and $R \cdot\left(a d+d^{2}\right)(R)=0$. Thus, the ideal of $R$ generated by $\left(a d+d^{2}\right)(R)$ is $\sum\left(a d+d^{2}\right)(R)$. Hence, $\sum\left(a d+d^{2}\right)(R) \cdot R=0$ implies $\sum\left(a d+d^{2}\right)(R)=0$. By Lemma $2,2 d(R)^{2}=0$, as desired.

Theorem 2. If $R$ is a prime ring with a derivation $d$ such that ax $+d(x) \epsilon$ $N \cap L$ for all $x$ in $R$, then either $R$ is associative or $\left(\left(a d+d^{2}\right)(R)\right)^{2}=0$.

Proof. Let $f=a d+d^{2}$. By the hypothesis, (5), (6) and (7), we have $f(R) \subseteq N \cap L$, $f(R) R \subseteq N \cap L$ and $R f(R) \subseteq N \cap L$. Applying these, and with $x \in f(R)$ and $y \in f(R)$, and with $x \in f(R)$ and $y \in f(R) R$, and with $x \in R f(R)$ and $y \in f(R)$ in (1) respectively, we all get $x y \in M$. Combining the above results, we obtain $f(R)^{2} \subseteq G, f(R) \cdot f(R) R \subseteq G$ and $R f(R) \cdot f(R) \subseteq G$. Using these and Lemma 1, we see that the ideal $C$ of $R$ generated by $f(R)^{2}$ is

$$
C=\sum f(R)^{2}+f(R)^{2} R+R f(R)^{2}+R \cdot f(R)^{2} R
$$

By (9), we have $C \cdot I=0$. Thus by the primeness of $R$, this implies $C=0$ or $I=0$. Hence either $R$ is associative or $\left(\left(a d+d^{2}\right)(R)\right)^{2}=0$, as desired. 
Theorem 3. If $R$ is a prime ring with a derivation d such that ax $+d(x) \in G$ for all $x$ in $R$, then either $R$ is associative or ad $+d^{2}=2 d(R)^{2}=0$.

Proof. Let $f=a d+d^{2}$. By the hypothesis, (5), (6) and (7), we get $f(R) \subseteq G$, $f(R) R \subseteq G$ and $R f(R) \subseteq G$. Using these and Lemma 1, we have that the ideal $F$ of $R$ generated by $f(R)$ is

$$
F=\sum f(R)+f(R) R+R f(R)+R \cdot f(R) R .
$$

By (9), we obtain $F \cdot I=0$. Thus by the primeness of $R$, this implies $F=0$ or $I=0$. Hence either $R$ is associative or $a d+d^{2}=0$. By Lemma $2,2 d(R)^{2}=0$. This completes the proof of Theorem 3 .

In the course of the proof of Theorem 3, we obtain the

Corollary. If $R$ is a semiprime ring with a derivation $d$ such that ax $+d(x) \in$ $G$ and $a d(x)+d^{2}(x) \in I$ for all $x$ in $R$, then $a d+d^{2}=2 d(R)^{2}=0$.

A simple calculation shows that $d(x, y, z))=(d(x), y, z)+(x, d(y), z)+(x, y, d(z))$ for all $x, y, z$ in $R$. Applying this we have that all the above associative subrings of $R$ are $d$-invariant. Thus $d(G) \subseteq G$. Hence we can give another proof of Theorem 3 .

Another proof of Theorem 3. For all $x, y$ in $R$, we have

$$
a(x y)+d(x y)=x(a y+d(y))+d(x) y=(a x+d(x)) y+x d(y) \in G .
$$

Then with $x \in G$, or $y \in G$ in this equality respectively, and using the hypothesis, and noting that $G$ is a subring of $R$, we obtain $d(x) y \in G$ and $x d(y) \in G$. Thus, we get $d(G) R+R d(G) \subseteq G$. Hence by Lemma 1, the ideal $H$ of $R$ generated by $d(G)$ is

$$
H=\sum d(G)+d(G) R+R d(G)+R \cdot d(G) R .
$$

Applying $d(G) \subseteq G$, (2), (8) and $d(G) R+R d(G) \subseteq G$, we have $d(G) \cdot I=0$. Thus, we get $H \cdot I=0$. By the primeness of $R$, this implies $H=0$ or $I=0$. If $I=0$, then $R$ is associative. Assume that $H=0$. Then $d(G)=0$ and so $\left(a d+d^{2}\right)(x)=a d(x)+d^{2}(x)=$ $d(a x+d(x))=0$ for all $x$ in $R$. Hence, $R$ is associative or $a d+d^{2}=0$.

Finally, we pose the following more general

Problem. If $R$ is a prime ring with a derivation $d$ and there exists a polynomial $f(t) \in \mathbb{Z}[t]$ such that $f(d)(x) \in G$ for all $x$ in $R$, then either $R$ is associative or $g(d)=0$ for some $g(t) \in \mathbf{Z}[t]$.

By Theorems A, B and 3, we know that this problem is true when $f(t)=t, t^{n}, a+t$, and $g(t)=2 t, t^{3 n-1}$ and $(a+t) t$ respectively. 
For the problem, we have the following remark by using the standard argument in linear algebra.

Remark. If $R$ is a nonassociative algebra over a field $F$ and $d$ is a derivation of $R$ such that $f(d)(R) \subseteq G$, where

$$
f(t)=\Pi_{i=1}^{n}\left(a_{i}+t\right)^{m_{i}} \in F[t], a_{i} \neq a_{j} \quad \text { if } i \neq j, i, j \in\{1,2, \cdots, n\},
$$

then $R=\sum_{i=1}^{n} R_{i}$ is a vector subspaces sum, where

$$
R_{i}=\left\{x \in R:\left(a_{i}+d\right)^{m_{i}}(x) \in G\right\}
$$

and

$$
R_{i} \cap \sum_{j \neq i_{j=1}}^{n} R_{j}=G, \quad i=1,2, \cdots, n .
$$

Moreover, if $m_{i}=1$ and $G R_{i}+R_{i} G \subseteq R_{i}, i=1,2, \cdots, n$, then as another proof of Theorem 3 we have

$$
d(G) R_{i}+R_{i} d(G) \subseteq G, i=1,2, \cdots, n,
$$

and so

$$
d(G) R+R d(G) \subseteq G
$$

Thus, if $R$ is prime then as above we can prove that $R$ is associative or $d(G)=0$ which implies $f(d) d=0$. For the general case, if $\operatorname{char}(R)=0$ then we may consider the tensor algebra $R \otimes_{F} E$, where $E$ is the algebraic closure of $F$.

We note that Theorem 3 can be generalized to skew derivation. Some lemmas and theorems of [6] have extended to $s$-derivations $d$ with $s d=d s$. The above results will be a part of my doctoral thesis at Taiwan University under the guidance of Professor Pjek-Hwee Lee. I thank him very much and I also thank my teacher Professor Tsiu-Kwen Lee. Finally, the author thanks the referee for careful comments.

\section{References}

[1] E. Kleinfeld, "A class of rings which are very nearly associative," Amer. Math. Monthly, 93(1986), 720-722.

[2] P. H. Lee and T. K. Lee, "Note on nilpotent derivations," Proc. Amer. Math. Soc., 98(1986), 31-32.

[3] T. I. Suh, "Prime nonassociative rings with a special derivation," Abstracts of papers presented to the Amer. Math. Soc., 14(1993), 284.

[4] C. T. Yen, "Rings with associators in the left and middle nucleus," Tamkang J. Math., 23(1992), 363-369.

[5] C. T. Yen, "Rings with associators in the nuclei," unpublished manuscript.

[6] C. T. Yen, "Rings with a derivation whose image is contained in the nuclei," Tamkang J. Math., 25(1994), 301-307.

[7] C. T. Yen, "Rings with a Jordan derivation whose image is contained in the nuclei or commutative center," submitted. 
[8] C. T. Yen, "Prime ring with a derivation whose some power image is contained in the nucleus," Soochow J. Math. to appear.

Department of Mathematics, Chung Yuan University, Chung Li, Taiwan 320, Republic of China. 